\title{
Monte Carlo Estimation of Time Mismatch Effect in an OFDM EER Architecture
}

\author{
J-F.Bercher, A.Diet, C.Berland, G.Baudoin, M.Villegas \\ jf.bercher@esiee.fr, a.diet@esiee.fr, c.berland@esiee.fr, g.baudoin@esiee.fr, m.villegas@esiee.fr \\ ESYCOM - ESIEE (communication system lab.): 2, Boulevard Blaise Pascal, Cité Descartes, BP99, 93162 Noisy-le-Grand - France
}

\begin{abstract}
New 5 GHz Wireless Local Area Networks standards uses OFDM modulation in order to increase data rate transfer. OFDM transmitter needs linearization technique due to non-linearities of the power amplification operation. EER architecture can be used to solve this problem while keeping a high efficiency. However several sources of imperfections can lower the quality of the signal. Time mismatch has especially a great impact. This paper presents a Monte Carlo study of envelope/phase delay influence on the OFDM signal. The Autocorrelation is estimated considering the OFDM signal as complex Gaussian.
\end{abstract}

\section{Introduction}

New $3^{\text {rd }}$ Generation standards such as Hiperlan2 or IEEE 802.11a uses OFDM (Orthogonal Frequency Division Multiplex) at $5 \mathrm{GHz}$. The advantages are a high data rate transfer and robustness in multi-paths environment. Each of the sub-carriers $(64$ for Hiperlan2) uses a QAM modulation scheme. The high disadvantage of OFDM is that the envelope of the emitted signal exhibits a large amplitude range. Consequently the power is un-constant which results in distortions caused by the non-linearities in the radiofrequency transmitter. Linearization methods are necessary. EER (Envelope Elimination and Restoration) introduced by Kahn in 1957 [1] is a solution to linearize the transmitter while keeping high efficiency. EER is based on the decomposition of the emitted signal in a magnitude signal (envelope varying) and a phase signal (constant power). Each signal is amplified separately. Recombination of the information is done by supply modulation of the high efficiency RF power amplifier (PA) as shown on Fig. 1.

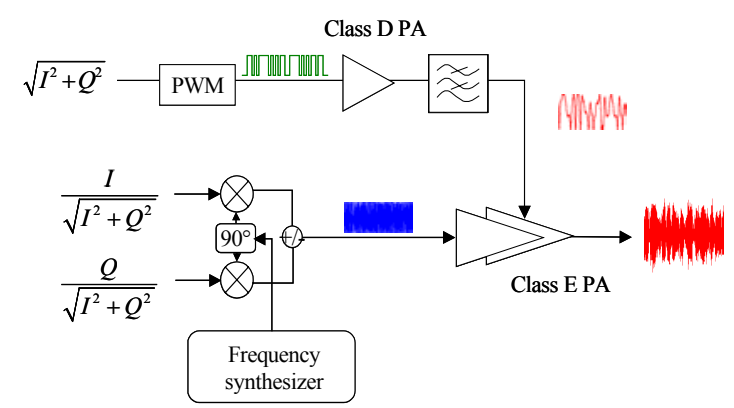

Fig. 1: EER architecture principle

In this architecture, several sources of imperfections lower the quality of the transmission [2]. The major impact is due to time mismatch $\Delta$ between envelope and phase signals at the recombination. This is caused by different operations on each path Consequences are noise and phase rotation [3]. A statistical Monte Carlo analysis enables a characterization of delay influence on the emitted signal.
The design of such architecture needs to precisely quantify the spectral effects of the previous defaults. A possible approach would be to study statistically the power spectrum obtained at the output of a simulated system, while varying values of the potential defaults. In fact, the impact of these defaults can be directly analysed using simulated signal generated with the same statistical properties as those of the potential outputs of the system. Hence, the results are obtained without resorting on a complete simulation of the whole system, that is interesting as far as computational load and simulation duration are concerned. It is also interesting in the fact that it focuses on the actual defaults under concern and not on other 'hidden' defaults on the simulation process.

\section{Simulations}

What we are ultimately interested in is the emitted spectrum. By Wiener-Kintchine theorem, it is the Fourier transform of the autocorrelation function $\operatorname{Rxx}(\tau)$. Because of time mismatch between envelope and phase components, the actual emitted signals is

$$
\begin{aligned}
& \mathrm{x}(\mathrm{t})=\rho(\mathrm{t}) \mathrm{e}^{\mathrm{j} \rho(\mathrm{t})}: \quad \text { OFDM signal } \\
& \mathrm{x}_{\Delta}(\mathrm{t})=\rho(\mathrm{t}) \cdot \mathrm{e}^{\mathrm{j} \varphi(\mathrm{t}-\Delta)} \quad: \quad \text { distorted signal }(\Delta)
\end{aligned}
$$

Hence, its autocorrelation writes:

$$
\begin{aligned}
& \operatorname{Rx}_{\Delta} \mathrm{x}_{\Delta}(\tau)=\mathrm{E}\left[\mathrm{x}_{\Delta}(\mathrm{t}) \mathrm{x}^{*}{ }_{\Delta}(\mathrm{t}-\tau)\right] \\
& \operatorname{Rx}_{\Delta} \mathrm{x}_{\Delta}(\tau)=\mathrm{E}\left[\rho(\mathrm{t}) \cdot \mathrm{e}^{\mathrm{j} \rho(\mathrm{t}-\Delta)} \rho(\mathrm{t}-\tau) \cdot \mathrm{e}^{-\mathrm{j} \varphi(\mathrm{t}-\Delta-\tau)}\right]
\end{aligned}
$$

where $\mathrm{E}[$.$] is the statistical average operator with the$ underlying probability distribution

$$
\mathrm{P}\{\rho(\mathrm{t}), \varphi(\mathrm{t}-\Delta), \rho(\mathrm{t}-\tau), \varphi(\mathrm{t}-\Delta-\tau)\}
$$

The Autocorrelation may be estimated as the time average:

$$
\hat{\mathrm{R}} \mathrm{x}_{\Delta} \mathrm{x}_{\Delta}(\tau)=\frac{1}{\mathrm{~N}} \sum_{\mathrm{i}=1}^{\mathrm{N}} \rho(\mathrm{i}-\tau) \cdot \mathrm{e}^{-\mathrm{j} \varphi(\mathrm{i}-\Delta-\tau)} \cdot \rho(\mathrm{i})^{\mathrm{j} \varphi(\mathrm{i}-\Delta)}
$$

from the output $\mathrm{x}_{\Delta}(\mathrm{t})$ of a simulated system.

The idea here is to estimate the autocorrelation (1) by ensemble average. This means that $\operatorname{Rx}_{\Delta} \mathrm{x}_{\Delta}(\tau)$ is computed as the mean of function of $\mathrm{K}$ independent realisations of 4 random variables $\left\{\rho_{1}, \varphi_{2}, \varphi_{3}, \rho_{4}\right\}$ with the correct statistics:

$$
\hat{\mathrm{R}} \mathrm{x}_{\Delta} \mathrm{x}_{\Delta}(\tau)=\frac{1}{\mathrm{~K}} \sum_{\mathrm{k}=1}^{\mathrm{K}} \rho_{1}(\mathrm{k}) \cdot \mathrm{e}^{-\mathrm{j} \varphi_{2}(\mathrm{k})} \cdot \rho_{4}(\mathrm{k})^{\mathrm{j} \varphi_{3}(\mathrm{k})}
$$

In the OFDM context, the emitted signal can be considered as complex Gaussian if the number of subcarrier (Nsub) is high enough. This hypothesis (Central Limit Theorem) is valid for Nsub greater than 30. For 
Hiperlan2 $(\mathrm{Nsub}=64)$, we model the OFDM signal as a Gaussian signal with known autocorrelation $\operatorname{Rxx}(\tau)$.

Hence, the random variables $\left\{\rho_{1}, \varphi_{2}, \varphi_{3}, \rho_{4}\right\}$ in (2) must have the same joint distribution as the variables $\{\rho(\mathrm{t}), \varphi(\mathrm{t}-\Delta), \varphi(\mathrm{t}-\tau-\Delta), \rho(\mathrm{t}-\tau)\}$ in $(1)$.

Equivalently, we shall generate a vector $\underline{\mathrm{W}}$,

$$
\underline{\mathrm{W}}=\left\{\mathrm{w}_{1}, \mathrm{w}_{2}, \mathrm{w}_{3}, \mathrm{w}_{4}\right\}
$$

with same joint distribution as a vector of samples of our OFDM signal $\underline{X}$ :

$$
\begin{gathered}
\underline{X}=\{\mathrm{x}(\mathrm{t}), \mathrm{x}(\mathrm{t}-\Delta), \mathrm{x}(\mathrm{t}-\Delta-\tau), \mathrm{x}(\mathrm{t}-\tau)\} \\
\underline{\mathrm{X}}=\left\{\rho(\mathrm{t}) \mathrm{e}^{\mathrm{i} \varphi(\mathrm{t})}, \rho(\mathrm{t}-\Delta) \mathrm{e}^{\mathrm{j} \varphi(\mathrm{t}-\Delta)}, \rho(\mathrm{t}-\Delta-\tau) \mathrm{e}^{\mathrm{j} \varphi(\mathrm{t}-\Delta-\tau)}, \rho(\mathrm{t}-\tau) \mathrm{e}^{\mathrm{j} \varphi(\mathrm{t}-\tau)}\right\}
\end{gathered}
$$

As $\underline{X}$, the samples of our OFDM signal, is a Gaussian vector, it suffice to impose that $\underline{\mathrm{W}}$ and $\underline{X}$ have the same correlation matrix. This correlation matrix is analytically known (since it depends on the known autocorrelation $\operatorname{Rxx}(\tau)$ ), and it is easy to generate $\underline{\mathrm{W}}$ as a simple transform of a random gaussian vector $\underline{\mathrm{G}}$ with uncorrelated components. The correlation matrix is given by:

$$
\underline{\mathrm{R}}_{\Delta, \tau}=\mathrm{E}\left[\underline{\mathrm{X}} \underline{X}^{*}\right]=\left(\begin{array}{cccc}
\operatorname{Rxx}(0) & \operatorname{Rxx}(\Delta) & \operatorname{Rxx}(\Delta+\tau) & \operatorname{Rxx}(\tau) \\
\operatorname{Rxx}(-\Delta) & \operatorname{Rxx}(0) & \operatorname{Rxx}(\tau) & \operatorname{Rxx}(-\Delta+\tau) \\
\operatorname{Rxx}(-\Delta-\tau) & \operatorname{Rxx}(-\tau) & \operatorname{Rxx}(0) & \operatorname{Rxx}(-\Delta) \\
\operatorname{Rx}(-\tau) & \operatorname{Rxx}(\Delta-\tau) & \operatorname{Rxx}(\Delta) & \operatorname{Rxx}(0)
\end{array}\right)
$$

(where $*$ denotes the hermitian transpose)

The key of the simulation process is to remark that given any square root $\underline{\underline{\mathrm{C}}}$ of $\underline{\underline{\mathrm{R}}}$ (i.e. a cholesky factor or a matrix of eigenvectors) such that $\underline{\underline{\mathrm{R}}}=\underline{\underline{\mathrm{CC}}}$, we have:

$$
\mathrm{E}[\underline{\mathrm{W}} \underline{\underline{\mathrm{W}}} *]=\mathrm{E}\left[\underline{\underline{\mathrm{CG}}} \underline{\mathrm{G}}^{*} \underline{\underline{\mathrm{C}}}^{*}\right]=\mathrm{E}[\underline{\underline{\mathrm{CC}}} *]=\underline{\underline{\mathrm{R}}}_{\Delta, \tau}
$$

with $\underline{\mathrm{W}}=\underline{\underline{\mathrm{CG}}}$ and $\underline{\mathrm{G}}$ a random zero mean Gaussian vector with uncorrelated components.

So, for a given $\Delta$, the simulation consist in:

- generate a Gaussian random vector $\underline{G}$ with uncorrelated components.

- compute $\underline{\mathrm{W}}=\underline{\underline{\mathrm{C}}} \underline{\mathrm{g}}$ for some values of $\tau$.

- update $\hat{\mathrm{R}} \mathrm{x}_{\Delta} \mathrm{x}_{\Delta}(\tau)$

Finally compute the spectrum by Fourier transform. Results of autocorrelation simulation are reported on Fig. 2 where the delay is varied from 0 to $10 \mathrm{nsec}$ considering a $20 \mathrm{MHz}$ OFDM signal using a root raised cosine shape filter (roll-off $=0.5$ ). Results show that delay causes small variations on the autocorrelation response.

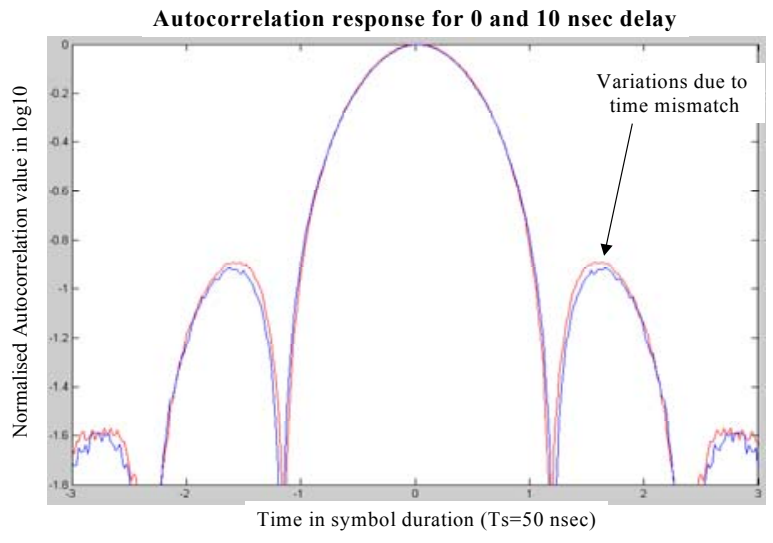

Fig. 2: Autocorrelation response from simulations
Resulting spectrums ( 1 to 10 nsec delay, 10 curves) show spectral re-growths. When compared to Hiperlan2 spectral limit, a delay of $5 \mathrm{nsec}$ is too high to fulfil standard requirements.

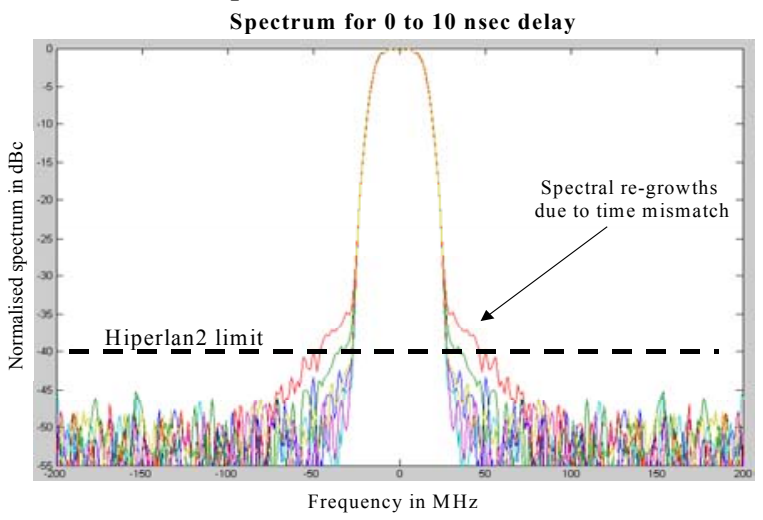

Fig. 3: Spectrum from simulated autocorrelations

Simulations are compared with HP-ADS Hiperlan2 ones (Fig. 4). Results showed in [2] a limit of 3 nsec. The difference is explained by the difference in spectrum calculation and non-ideal Gaussian behaviour of Hiperlan2 simulated signal. The accuracy can be improved by increasing K (10000 here). Confidence intervals can be computed using Student tests.

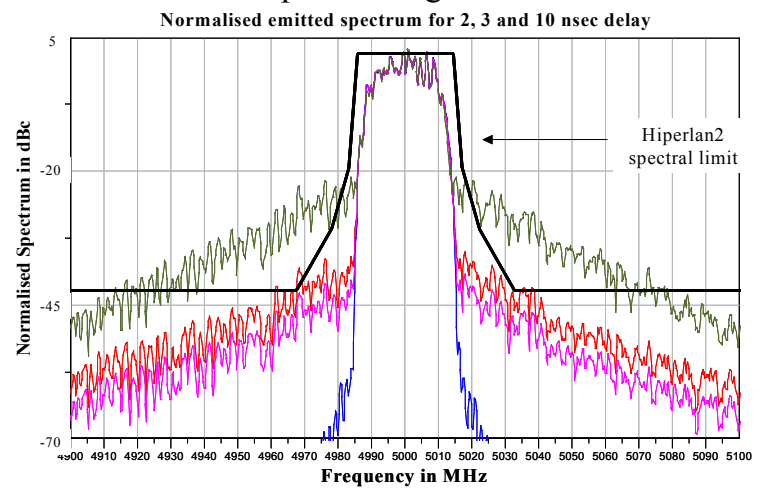

Fig. 4: Simulated spectrum with HP-ADS

\section{Conclusion}

It was presented an estimation of envelope/phase time mismatch influence on an OFDM signal. This is particularly important in a sensibility analysis of EER architecture. Results showed a good agreement between simulated Monte Carlo study results and HPADS Hiperlan2 simulation ones. Characterizing the autocorrelation of the envelope delayed OFDM signal is possible. With simulated spectrums values, the delay imperfection can be analysed, and the impact of delay imperfection can be investigated.

\section{References}

[1] L.Kahn, "Single sideband transmission by envelope elimination and restoration", IRE proc., pp 803-806, July 1952.

[2] A.Diet, C.Berland, M.Villegas, G.Baudoin, "Sensibilité d'une architecture EER à une modulation OFDM dans le système Hiperlan2", XIIIèmes journées nationales micro-ondes à Lille, 2003. [3] G.Baudoin, C.Berland, M.Villegas, A.Diet, "Influence of time and processing mismatches between phase and envelope in EER". IEEE, International Microwave symposium, 8-13 June 2003 Philadelphia, Pennsylvania, USA

[4] J.Proakis "Digital Communications". McGrawHill Edition, Boston, fourth edition, 2001. 